\title{
Velocity-Free Adaptive Time Delay Control of Robotic System
}

\author{
Shini Chen ${ }^{1,2}$ and Xia Liu $\mathbb{D}^{1,2}$ \\ ${ }^{1}$ School of Electrical Engineering and Electronic Information, Xihua University, Chengdu 610039, China \\ ${ }^{2}$ Centre for Robotics Research, Xihua University, Chengdu 610039, China \\ Correspondence should be addressed to Xia Liu; xliu_uestc@yahoo.com
}

Received 18 July 2020; Revised 22 October 2020; Accepted 11 November 2020; Published 24 November 2020

Academic Editor: Raúl Villafuerte-Segura

Copyright (c) 2020 Shini Chen and Xia Liu. This is an open access article distributed under the Creative Commons Attribution License, which permits unrestricted use, distribution, and reproduction in any medium, provided the original work is properly cited.

\begin{abstract}
To improve the trajectory tracking performance of a complex nonlinear robotic system, a velocity-free adaptive time delay control is proposed. First, considering that conventional time delay control (TDC) may cause large time delay estimation (TDE) error under nonlinear friction, a TDC with gradient estimator is designed. Next, since it is complicated and time-consuming to adjust gains manually, an adaptive law is designed to estimate the gain of the gradient. Finally, in order to avoid the measurement of velocity and acceleration in the controller while enabling the robot to implement position tracking, an observer is designed. The proposed control can not only offset the nonlinear terms in the complex dynamics of the robotic system but also reduce the TDE error, estimate the gain of the gradient online, and avoid the measurement of velocity and acceleration. The stability of the system is analyzed via Lyapunov function. Simulations are conducted on a 2-DOF robot to verify the effectiveness of the proposed control.
\end{abstract}

\section{Introduction}

Robotic system is a nonlinear and strongly coupled system subject to various uncertainties, such as unmodeled dynamics and nonlinear friction [1]. These nonlinearity and uncertainties may degrade the tracking performance of the robotic system and even make the system unstable [2, 3].

In order to guarantee the tracking performance of robotic system with unknown dynamics, time delay control (TDC) which has a simple structure and good robustness was proposed; this was pioneered by Youcef-Toumi and Ito [4]. The main idea of TDC is to employ time delay estimation (TDE) technology. In TDE, the time delay of input torque and acceleration is employed to estimate the complex unknown dynamics and nonlinear terms which are difficult to handle. However, since the nonlinear terms are divided into soft nonlinearity and hard nonlinearity such as discontinuous Coulomb friction and static friction $[5,6]$, TDC has some inherent limitations. In fact, Coulomb friction accounts for $30 \%$ of the maximum control torque and cannot be ignored $[7,8]$, which results in large TDE error and greatly affects the system performance.
In order to compensate the TDE error caused by TDC, many auxiliary controllers have been designed. In [9], an adaptive gain sliding mode TDC method was designed for robot manipulators. The sliding mode control was used to reduce the TDE error, and the adaptive law was employed to reduce the chattering near the sliding mode surface. Based on the work in [9], a wide adaptive gain sliding mode TDC method was designed in [10]. The wide adaptive law was used to make the control gain range wider and suppress the adverse TDE error. The tracking performance and robustness of the system were improved. In [11], an adaptive TDC was designed for cable-driven manipulators to suppress the chattering and the TDE error. A fractional-order and nonsingular sliding mode surface was used to introduce the desired error dynamics. Moreover, the continuous and chatter-free adaptive gains were used to enhance the control performance under time-varying disturbances. In [12], a TDC method with ideal velocity feedback was designed for a robot manipulator with nonlinear friction. The ideal velocity feedback was used to cancel the hard nonlinearity which cannot be cancelled by TDC, suppressing the TDE error. The controller has a simple structure and yet provides good 
online friction compensation. In [13], an inclusive enhanced TDC with nonlinear desired error dynamics was designed and the nonlinear sliding mode surface was used to reduce the TDE error and chattering. The approach inherits the advantages of TDC that is simple and model-free. In [14], a TDC method with internal model was designed for robot manipulators, where the internal model played a role in compensating for hard nonlinear friction and eliminating disturbance. The controller has a synergy effect coming from the complementary use of TDC and internal model. In [15], a fuzzy logic TDC was designed for a cable-driven robot. The TDC was used to estimate and cancel the soft nonlinearity while the fuzzy logic was used to cancel the hard nonlinearity. The fuzzy logic TDC not only has a simple structure but also can effectively track the desired trajectory. Although the TDE error can be offset effectively by the control methods in [9-15], several gains need to be adjusted and the velocity and acceleration need to be measured additionally. Since it is a time-consuming task to adjust gains, a TDC method based on gradient estimator was designed for robot manipulator in [16]. The TDE error can be effectively suppressed by the gradient estimator, and only one gain needs to be adjusted additionally. However, the gain of the gradient in [16] needs to be adjusted manually, and the velocity and acceleration in the controller still need to be measured, which may easily result in measurement noise.

Therefore, this paper presents a velocity-free adaptive TDC to improve the trajectory tracking performance of complex nonlinear robotic system. The main contributions of this paper are as follows: (1) a TDC with gradient estimator is designed to reduce the TDE error in the conventional TDC; (2) an adaptive law is designed to estimate the gain of the gradient online; and (3) an observer is designed to observe the velocity and acceleration in the controller, which can avoid the measurement of velocity and acceleration.

This paper is organized as follows. Section 2 states the problems of the conventional TDC. In Section 3, velocityfree adaptive TDC is presented. In Section 4, stability of the system is proved using Lyapunov stability synthesis. Comparative simulations are conducted to validate the proposed control in Section 5. Section 6 concludes the paper.

\section{Problem Statement}

The general dynamics of a $n$-DOF robot can be considered as follows [17]:

$$
\boldsymbol{\tau}=\mathbf{M}(\mathbf{q}) \ddot{q}+\mathbf{C}(\mathbf{q}, \dot{q}) \dot{q}+\mathbf{G}(\mathbf{q})+\mathbf{f},
$$

where $\tau \in \mathbf{R}^{n \times 1}$ is the input torque of the robot, $\mathbf{M}$ is the positive inertia matrix, $\mathbf{C}(\mathbf{q}, \dot{q}) \in \mathbf{R}^{n \times n}$ is the centripetal and Coriolis matrix, $\mathbf{G}(\mathbf{q}) \in \mathbf{R}^{n \times 1}$ is the gravity term, $\mathbf{f} \in \mathbf{R}^{n}$ is the friction torque, and $\mathbf{q} \in \mathbf{R}^{n \times 1}, \dot{q} \in \mathbf{R}^{n \times 1}$, and $\ddot{q} \in \mathbf{R}^{n \times 1}$ are the angular position, velocity, and acceleration of the robot, respectively.

Introducing a positive-definite diagonal constant matrix $\bar{M}$ into the general dynamics (1) of the robot, we can get

$$
\bar{M} \ddot{q}+\mathbf{N}(\mathbf{q}, \dot{q}, \ddot{q})=\boldsymbol{\tau},
$$

where $\quad \mathbf{N}(\mathbf{q}, \dot{q}, \ddot{q}) \triangleq[\mathbf{M}(\mathbf{q})-\bar{M}] \ddot{q}+\mathbf{C}(\mathbf{q}, \dot{q}) \dot{q}+\mathbf{G}(\mathbf{q})+\mathbf{f}$, which contains all the nonlinear terms in the complex dynamics of the robotic system. In practice, since $\mathbf{N}(\mathbf{q}, \dot{q}, \ddot{q})$ is very complicated and difficult to calculate, the main idea of conventional TDC is to employ TDE [18] to get its estimation $\widehat{N}(\mathbf{q}, \dot{q}, \ddot{q})$ :

$$
\mathbf{N}(\mathbf{q}, \dot{q}, \ddot{q}) \approx \mathbf{N}(\mathbf{q}, \dot{q}, \ddot{q})_{t-L} \triangleq \widehat{N}(\mathbf{q}, \dot{q}, \ddot{q}) .
$$

Notice that $L$ represents the time delay. Obviously, when $L$ is small enough, $\mathbf{N}(\mathbf{q}, \dot{q}, \ddot{q})$ can be accurately estimated by (3). The term $\mathbf{N}(\mathbf{q}, \dot{q}, \ddot{q})_{t-L}$ intentionally introduces the time delay $L$ to estimate $\mathbf{N}(\mathbf{q}, \dot{q}, \ddot{q})$. According to (2), the complex dynamics of the robot can be estimated by $\mathbf{N}(\mathbf{q}, \dot{q}, \ddot{q})_{t-L}$ which is a simple structure.

Substituting (3) into (2), we can get the TDE as

$$
\mathbf{N}(\mathbf{q}, \dot{q}, \ddot{q})_{t-L}=\boldsymbol{\tau}_{t-L}-\bar{M} \ddot{q}_{t-L} .
$$

Let the desired error dynamics of the system be

$$
\ddot{e}+\mathbf{K}_{D} \dot{e}+\mathbf{K}_{p}=0,
$$

where $\mathbf{e}=\mathbf{q}_{d}-\mathbf{q}$ is the position tracking error of the robot.

Rearranging (2)-(5), we can get the conventional TDC as

$\boldsymbol{\tau}=\underbrace{\boldsymbol{\tau}_{t-L}-\bar{M} \ddot{q}_{t-L} T D E}_{\text {desired error dynamics }}+\bar{M} \underbrace{\left[\ddot{q}_{d}+K_{D}\left(\dot{q}_{d}-\dot{q}\right)+K_{P}\left(q_{d}-q\right)\right]}$,

where $\mathbf{q}_{d}, \dot{q}_{d}$, and $\ddot{q}_{d}$ denote the desired position, velocity, and acceleration of the robot and $\mathbf{K}_{D}$ and $\mathbf{K}_{P}$ represent the diagonal gain matrices of decoupled PD controllers. The first two terms $\tau_{t-L}-\bar{M} \ddot{q}_{t-L}$ consist of the TDE which offsets $\mathbf{N}(\mathbf{q}, \dot{q}, \ddot{q})$, and the other terms inject the desired error dynamics.

Remark 1. The term $\mathbf{N}(\mathbf{q}, \dot{q}, \ddot{q})$ in (2) contains all the nonlinear terms including uncertain parameters and friction of the robotic system. Since $\mathbf{N}(\mathbf{q}, \dot{q}, \ddot{q})$ can be offset by $\mathbf{N}(\mathbf{q}, \dot{q}, \ddot{q})_{t-L}$ in (3), the uncertain parameters and friction have no effect on the dynamic response of the control system.

From (3), we can see that when $L$ is small enough, $\mathbf{N}(\mathbf{q}, \dot{q}, \ddot{q})$ can be accurately estimated. However, in practice, the minimum value of $L$ can only be the sampling time. This limitation on $L$ will cause TDE error. From (3), (4), and (6), we can get

$$
\begin{aligned}
\mathbf{N}(\mathbf{q}, \dot{q}, \ddot{q})-\widehat{N}(\mathbf{q}, \dot{q}, \ddot{q}) & =\mathbf{N}(\mathbf{q}, \dot{q}, \ddot{q})-\mathbf{N}(\mathbf{q}, \dot{q}, \ddot{q})_{t-L} \\
& =\bar{M}\left[\ddot{e}+\mathbf{K}_{D} \dot{e}+\mathbf{K}_{P} \mathbf{e}\right] .
\end{aligned}
$$

From (5) and (7), we can obtain the TDE error $\varepsilon$ :

$$
\boldsymbol{\varepsilon} \triangleq \bar{M}^{-1}\left[N(\mathbf{q}, \dot{q}, \ddot{q})-N(\mathbf{q}, \dot{q}, \ddot{q})_{t-L}\right]=\ddot{e}+\mathbf{K}_{D} \dot{e}+\mathbf{K}_{P} \mathbf{e} .
$$

Obviously, there exits a deviation between $\varepsilon$ and the desired error dynamics (5). Especially when there exists hard nonlinear friction such as Coulomb friction and static friction, the TDE error will become very large, affecting the tracking performance of the robotic system. 


\section{Velocity-Free Adaptive TDC}

The idea of the proposed velocity-free adaptive TDC of robotic system is shown in Figure 1. TDC with gradient estimator $\tau$ is designed to reduce the TDE error $\varepsilon$. An adaptive law is designed to estimate the gain of the gradient and obtain its estimation $\widehat{K}_{\mathrm{GE}}$. An observer is designed to observe the velocity and acceleration and obtain the estimation of velocity $\hat{x}_{1}$ and the estimation of acceleration $\dot{\hat{x}}_{2}$, respectively. In this way, the real position $\mathbf{q}$ of the robot can track the desired position $\mathbf{q}_{d}$.

3.1. Design of TDC with Adaptive Gradient Estimator. Introducing the term $\widehat{\varepsilon}$ of gradient estimator into the TDC (6), we can get the TDC with gradient estimator as

$$
\boldsymbol{\tau}=\boldsymbol{\tau}_{t-L}-\bar{M} \ddot{q}_{t-L}+\bar{M}\left[\ddot{q}_{d}+\mathbf{K}_{D}\left(\dot{q}_{d}-\mathbf{q}\right)+\mathbf{K}_{P}\left(\mathbf{q}_{d}-\mathbf{q}\right)\right]+\bar{M} \widehat{\varepsilon} .
$$

Substituting (9) into (2), we can obtain the closed-loop dynamics error of TDC with gradient estimator:

$$
\ddot{e}+K_{D} \dot{e}+\mathbf{K}_{P} \mathbf{e}=-\widetilde{\mathcal{E}},
$$

where $\widetilde{\varepsilon} \triangleq \widehat{\varepsilon}-\varepsilon$ represents the estimation error of the TDE error. Define the cost function of the estimation error as

$$
\mathbf{J}(\widetilde{\mathcal{\varepsilon}})=\frac{1}{2} \widetilde{\mathcal{\varepsilon}} T \widetilde{\varepsilon} .
$$

Then, when the TDE error was slow-varying or constant, the gradient estimator can be designed as

$$
\dot{\vec{\varepsilon}}=-\mathbf{K}_{\mathrm{GE}} \frac{\partial \mathbf{J}}{\partial \widehat{\mathcal{\varepsilon}}}=-\mathbf{K}_{\mathbf{G E}} \widetilde{\varepsilon}=\mathbf{K}_{\mathbf{G E}}\left(\ddot{e}+\mathbf{K}_{D} \dot{e}+\mathbf{K}_{P} \mathbf{e}\right),
$$

where $\mathbf{K}_{\mathrm{GE}} \triangleq \operatorname{diag}\left(\mathrm{K}_{\mathrm{GE}_{1}}, \ldots, \mathrm{K}_{\mathrm{GE}_{n}}\right)$ is the positive gain matrix of the gradient estimator. Since the gradient estimator (12) always make the cost function surface negative, the gradient estimator can reduce the TDE error caused by TDC.

Therefore, (9) can be rewritten as

$$
\begin{aligned}
\boldsymbol{\tau}= & \underbrace{\boldsymbol{\tau}_{t-L}-\bar{M} \ddot{q}_{t-L}}_{\mathrm{TDE}}+\bar{M} \underbrace{\left[\ddot{q}_{d}+\mathbf{K}_{D}\left(\dot{q}_{d}-\dot{q}\right)+\mathbf{K}_{P}\left(\mathbf{q}_{d}-\mathbf{q}\right)\right]}_{\text {desired error dynamics }} \\
& +\underbrace{\bar{M} \mathbf{K}_{\mathbf{G E}}\left(\dot{e}+\mathbf{K}_{D} \mathbf{e}+\mathbf{K}_{P} \int \mathbf{e d t}\right)}_{\text {gradient estimator }} .
\end{aligned}
$$

It can be seen from (13) that the TDC with gradient estimator does not need the nonlinear terms in the complex dynamics of the robotic system and can reduce the TDE error. In addition, the TDC with gradient estimator (13) only needs to adjust one gain, i.e., the gain of the gradient $\mathrm{K}_{\mathrm{GE}}$, assuming $\mathbf{K}_{P}=\beta \mathbf{K}_{S}, \mathbf{K}_{D}=\mathbf{K}_{S}+\beta(\beta>0), \mathbf{S}=\dot{e}+\mathbf{K}_{S} \mathbf{e}$, and $\mathbf{K}_{S}$ is a positive diagonal constant matrix. In order to estimate $\mathbf{K}_{\mathrm{GE}}$, the following adaptive law is designed:

$$
\dot{\hat{K}}_{\mathrm{GE}}=\frac{\psi}{\alpha}\|\mathbf{S}\|-\boldsymbol{\varepsilon},
$$

where $\alpha$ and $\psi$ are positive parameters and $\varepsilon=\ddot{e}+\mathbf{K}_{D} \dot{e}+\mathbf{K}_{P} \mathbf{e}$.

According to (13) and (14), we can design the TDC with adaptive gradient estimator as

$$
\begin{aligned}
\boldsymbol{\tau}= & \underbrace{\boldsymbol{\tau}_{t-L}-\bar{M} \ddot{q}_{t-L}}_{\mathrm{TDE}}+\bar{M} \underbrace{\left[\ddot{q}_{d}+\mathbf{K}_{D}\left(\dot{q}_{d}-\dot{q}\right)+\mathbf{K}_{P}\left(\mathbf{q}_{d}-\mathbf{q}\right)\right]}_{\text {desired error dynamics }} \\
& +\underbrace{\bar{M} \widehat{K}_{\mathrm{GE}}\left(\dot{e}+\mathbf{K}_{D} \mathbf{e}+\mathbf{K}_{P} \int \mathbf{e d t}\right)}_{\text {adaptive gradient estimator }} .
\end{aligned}
$$

where $\tau_{t-L}-\bar{M} \ddot{q}_{t-L}$ is the TDE which offsets $\mathbf{N}(\mathbf{q}, \dot{q}, \ddot{q})$, $\bar{M}\left[\ddot{q}_{d}+\mathbf{K}_{D}\left(\dot{q}_{d}-\dot{q}\right)+\mathbf{K}_{P}\left(\mathbf{q}_{d}-\mathbf{q}\right)\right]$ is the desired error dynamics, and $\bar{M} \widehat{K}_{\mathrm{GE}}\left(\dot{e}+t \mathbf{K}_{D} n e q+h \mathbf{K}_{P} \mathbf{e} \mathbf{d t}\right.$ is the adaptive gradient estimator to reduce the TDE error caused by TDC.

3.2. Design of the Observer. Let $\mathbf{x}_{1}=\mathbf{q}, \bar{x}_{2}=\dot{q}$. From (2) and (3), we can get the state-space equation of the dynamics of the robot (1):

$$
\left\{\begin{array}{l}
\dot{x}_{1}=\bar{x}_{2}, \\
\dot{\bar{x}}_{2}=-\bar{M}^{-1}\left[\mathbf{N}\left(\mathbf{x}_{1}, \bar{x}_{2}, \dot{\bar{x}}_{2}\right)_{t-L}-\tau_{t-L}\right] .
\end{array}\right.
$$

Then, define a new variable $\mathbf{x}_{2}=\bar{x}_{2}-\mathbf{T} \mathbf{x}_{1}$, where $\mathbf{T}=\operatorname{diag}\left(t_{1}, t_{2}, \ldots, t_{n}\right), t_{i}>0 \quad(i=1,2, \ldots, n)$. Substituting $\mathbf{x}_{2}$ into (16) gives

$$
\left\{\begin{array}{l}
\dot{x}_{1}=\mathbf{T} \mathbf{x}_{1}+\mathbf{x}_{2}, \\
\dot{x}_{2}=-\bar{M}^{-1}\left[\mathbf{N}\left(\mathbf{x}_{1}, \bar{x}_{2}, \dot{\bar{x}}_{2}\right)_{t-L}-\tau_{t-L}\right]-\mathbf{T x}_{2}-\mathbf{T}^{2} \mathbf{x}_{1} .
\end{array}\right.
$$

Suppose $\quad \mathbf{L}_{1}=\operatorname{di} \operatorname{ag}\left(l_{11}, l_{12}, \ldots, l_{1 n}\right), \quad \mathbf{L}_{2}=\mathbf{d i} \mathbf{a g}\left(l_{21}, l_{22}\right.$, $\left.\ldots, l_{2 n}\right)$, and $\mathbf{L}_{1}$ and $\mathbf{L}_{2}$ are positive-definite matrices. For simplicity, let $-\bar{M}^{-\mathrm{r}}\left[\mathbf{N}\left(\mathbf{x}_{1}, \bar{x}_{2}, \dot{\bar{x}}_{2}\right)_{t-L}-\tau_{t-L}\right]=\mathbf{F}\left(\mathbf{x}_{1}, \mathbf{x}_{2}\right)$. Now, the observer of the robotic system can be designed as

$$
\left\{\begin{array}{l}
\dot{\hat{x}}_{1}=\mathbf{T} \hat{x}_{1}+\hat{x}_{2}-\mathbf{L}_{1} \mathbf{e}_{x 1}+\mathbf{v}_{1}, \\
\dot{\hat{x}}_{2}=\mathbf{F}\left(\mathbf{x}_{1}, \hat{x}_{2}\right)-\mathbf{T} \hat{x}_{2}-\mathbf{T}^{2} \widehat{x}_{1}-\mathbf{L}_{2} \mathbf{e}_{x 2}+\mathbf{v}_{2}+\chi \operatorname{sign}\left(\mathbf{v}_{1}\right),
\end{array}\right.
$$

where $\mathbf{v}_{1}=-k_{1} \operatorname{sign}\left(\mathbf{e}_{1}\right), \mathbf{v}_{2}=k_{2} \mathbf{v}_{1}+k_{3}\left|\mathbf{v}_{1}\right|^{\gamma} \operatorname{sign}\left(\mathbf{v}_{1}\right), k_{1}, k_{2}$, $k_{3}>0,0<\Upsilon<1$, and $\chi$ is a positive-definite diagonal matrix. $\widehat{\mathbf{x}}_{1}$ and $\widehat{\mathbf{x}}_{2}$ are the estimations of $\mathbf{x}_{1}$ and $\mathbf{x}_{2}$, and $\mathbf{e}_{x 1}$ and $\mathbf{e}_{x 2}$ are the estimation errors of $\mathbf{x}_{1}$ and $\mathbf{x}_{2}$, respectively:

$$
\begin{aligned}
& \mathbf{e}_{x 1}=\hat{x}_{1}-\mathbf{x}_{1}, \\
& \mathbf{e}_{x 2}=\hat{x}_{2}-\mathbf{x}_{2} .
\end{aligned}
$$

From (18), it can be seen that the velocity $\dot{q}$ and acceleration $\ddot{q}$ of the robot system can be estimated by the observer. Consequently, the velocity and acceleration do not need to be measured.

Remark 2. In (19), $\mathbf{e}_{x 2}=\widehat{x}_{2}-\mathbf{x}_{2}$, where $\mathbf{x}_{2}$ is the velocity. Then, we can get $\dot{e}_{x 2}=\dot{\hat{x}}_{2}-\dot{x}_{2}$, where $\dot{x}_{2}$ is the acceleration $\ddot{q}$. Moreover, from (2), we have $\ddot{q}=\bar{M}^{-1}[\tau-\mathbf{N}(\mathbf{q}, \dot{q}, \ddot{q})]$. 


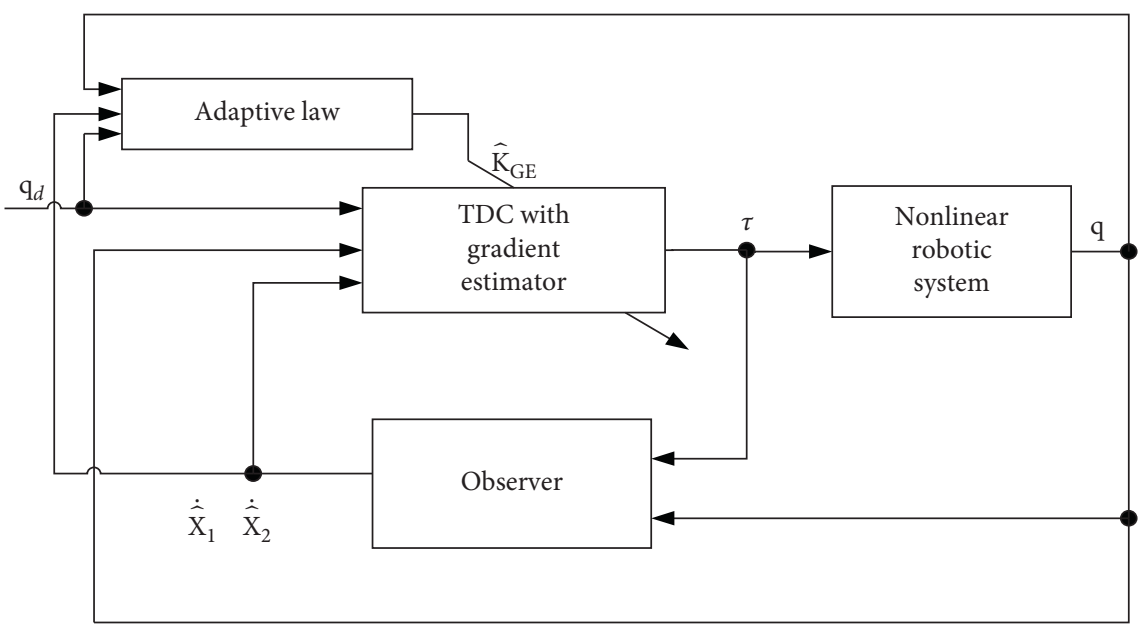

FIGURE 1: Block diagram of velocity-free adaptive TDC.

Therefore, we can avoid the direct measurement of the From (19), we can get the observer error as velocity in (18).

$$
\left\{\begin{array}{l}
\dot{e}_{x 1}=\left(\mathbf{T}-\mathbf{L}_{1}\right) \mathbf{e}_{x 1}+\mathbf{e}_{x 2}+\mathbf{v}_{1}, \\
\dot{e}_{x 2}=-\left(\mathbf{T}+\mathbf{L}_{2}\right) \mathbf{e}_{x 1}-\mathbf{T} \mathbf{e}_{x 2}+\mathbf{v}_{2}+\mathbf{F}\left(\mathbf{x}_{1}, \hat{x}_{2}\right)-\mathbf{F}\left(\mathbf{x}_{1}, \mathbf{x}_{2}\right)+\chi \operatorname{sign}\left(\mathbf{v}_{1}\right) .
\end{array}\right.
$$

Since the constant matrix $\mathbf{T}$ is easily adjusted in (18), the design is flexible. When the states $\dot{\hat{x}}_{1}$ and $\dot{\hat{x}}_{2}$ of the observer are far from the real states $\mathbf{x}_{1}$ and $\mathbf{x}_{2}$, the term $k_{2} \mathbf{v}_{1}$ can guarantee the rapid convergence of $\dot{e}_{x 1}$ and $\dot{e}_{x 2}$. Otherwise, the term $k_{3}\left|\mathbf{v}_{1}\right|^{\gamma} \operatorname{sign}\left(v_{1}\right)$ can guarantee the rapid convergence of $\dot{e}_{x 1}$ and $\dot{e}_{x 2}$.

Now, based on the TDC with adaptive gradient estimator and the observer, the velocity-free TDC with adaptive gradient estimator can be designed as

$$
\boldsymbol{\tau}=\underbrace{\boldsymbol{\tau}_{t-L}-\bar{M} \dot{\hat{x}}_{2 t-L}}_{\text {T DE }}+\bar{M} \underbrace{\left[\ddot{q}_{d}+\mathbf{K}_{D}\left(\dot{q}_{d}-\dot{\hat{x}}_{1}\right)+\mathbf{K}_{P}\left(\mathbf{q}_{d}-\mathbf{q}\right)\right]}_{\text {desired error dynamics }}+\underbrace{\bar{M} \widehat{K}_{\mathbf{G E}}\left(\dot{e}+\mathbf{K}_{D} \mathbf{e}+\mathbf{K}_{P} \int \mathbf{e d t}\right)}_{\text {adaptive gradient estimator }} .
$$

The controller (21) adopts the TDE $\tau_{t-L}-\bar{M} \dot{\hat{x}}_{2 t-L}$ which does not need the nonlinear terms in the complex dynamics of the robotic system. Meanwhile, $\bar{M}\left[\ddot{q}_{d}+\mathbf{K}_{D}\left(\dot{q}_{d}-\dot{\hat{x}}_{1}\right)+\right.$ $\left.\mathbf{K}_{P}\left(\mathbf{q}_{d}-\mathbf{q}\right)\right]$ injects the desired error dynamics and $\bar{M} \widehat{K}_{\mathrm{GE}}\left(\dot{e}+t \mathbf{K}_{D} n e q+h \mathbf{K}_{P} \mathbf{e} \mathbf{d t}\right.$ can reduce the TDE error caused by TDC using the adaptive gradient estimator. In addition, the use of observer can avoid the measurement noise of the velocity and acceleration in the robotic system.

Remark 3. In practice, there may still be the noise in the measurement of position. However, the influence of the noise caused by the measurement of position is far less than that of velocity and acceleration. The controller (21) can effectively avoid the measurement of velocity and acceleration.

\section{Stability Analysis}

4.1. Stability Analysis of the Observer. Let $\mathbf{F}\left(\mathbf{x}_{1}, \mathbf{x}_{2}\right), \mathbf{F}\left(\mathbf{x}_{1}, \widehat{x}_{2}\right)$ be the Lipschitz function [19], i.e., $\left\|\mathbf{F}\left(\mathbf{x}_{1}, \widehat{x}_{2}\right)-\mathbf{F}\left(\mathbf{x}_{1}, \mathbf{x}_{2}\right)\right\| \leq \bar{C}$, where $\bar{C}$ is a positive constant. Let us define $\mathbf{e}_{x}=\left[\mathbf{e}_{x 1}, \mathbf{e}_{x 2}\right]^{T}$. Then, from (5), we can get

$$
\dot{e}_{x}=\mathbf{A e}_{x}+\mathbf{F}+\bar{v}
$$

where $\mathbf{A}=\left[\begin{array}{cc}\mathbf{T}-\mathbf{L}_{1} & \mathbf{E} \\ -\mathbf{T}^{2}-\mathbf{L}_{2} & -\mathbf{T}\end{array}\right], \Delta \mathbf{F}=\left[\begin{array}{c}0_{\mathbf{n} \times 1} \\ \mathbf{F}\left(\mathbf{x}_{1}, \widehat{x}_{2}\right)-\mathbf{F}\left(\mathbf{x}_{1}, \mathbf{x}_{2}\right)\end{array}\right]$, and $\bar{v}=\left[\begin{array}{c}\mathbf{v}_{1} \\ \mathbf{v}_{2}+\chi \operatorname{sign}\left(\mathbf{v}_{1}\right)\end{array}\right]$.

Lemma 1 (see [20]). When the following equation holds, the observer error $\mathbf{e}_{x}$ will converge to zero in finite time: 


$$
\left\{\begin{array}{l}
\mathbf{P A}+\mathbf{A}^{T} \mathbf{P}+\frac{\mathbf{P P}}{\sigma}+\varphi<0, \\
l_{1 i}>t_{i}, \\
k_{1}-R=\eta>0, \\
-2 \mathbf{P}_{2} \mathbf{T}+\frac{\mathbf{P}_{2} \mathbf{P}_{2}}{\sigma}+\sigma \bar{C}_{2} \mathbf{E}<0,
\end{array}\right.
$$

where $P=\operatorname{diag}\left\{P_{1}, P_{2}\right\}>0, \quad \mathbf{P}_{1}=\operatorname{diag}\left\{p_{11}, p_{12}, \ldots, p_{1 n}\right\}$, $\mathbf{P}_{2}=\operatorname{di} \operatorname{ag}\left\{p_{21}, p_{22}, \ldots, p_{2 n}\right\}, \sigma, \eta, \overline{\mathbf{C}}>0, \varphi=\operatorname{diag}\left\{0_{\mathbf{n} \times \mathbf{n}}\right.$, $\left.\sigma \bar{C}^{2} \mathbf{E}\right\}$, and $\mathbf{R}$ is the boundary of $\left\|\mathbf{e}_{x}\right\|$.

Consider a Lyapunov function:

$$
\mathbf{V}_{1}=\mathbf{e}_{x}^{T} \mathbf{P} \mathbf{e}_{x} .
$$

Differentiating (24) with respect to time and substituting (22) and (23) into it yields

$$
\dot{V}_{1} \leq \mathbf{e}_{x}^{T}\left(\mathbf{P A}+\mathbf{A}^{T} \mathbf{P}\right) \mathbf{e}_{\mathbf{x}}+2 \mathbf{e}_{x}^{T} \mathbf{P F}+2 \mathbf{e}_{x}^{T} \mathbf{P} \bar{v} .
$$

According to (23) and (25), we have $2 \mathbf{e}_{x}^{T} \mathbf{P} \Delta \mathbf{F} \leq$ $\left(\left(\mathbf{e}_{x}^{T} \mathbf{P} \mathbf{P} \mathbf{e}_{x}\right) / \sigma\right)+\sigma \Delta \mathbf{F}^{T} \Delta \mathbf{F}$. Moreover, from (22), we know that $\mathbf{F}\left(\mathbf{x}_{1}, \widehat{x}_{2}\right), \mathbf{F}\left(\mathbf{x}_{1}, \mathbf{x}_{2}\right)$ is the Lipschitz function. Thus, we have $\mid \Delta \mathbf{F}^{T} \Delta \mathbf{F} \leq \bar{C}^{2}\left\|\mathbf{e}_{x 2}\right\|^{2}$. Consequently, (25) can be rewritten into

$$
\begin{aligned}
\dot{V}_{1} & \leq \mathbf{e}_{x}^{T}\left(\mathbf{P A}+\mathbf{A}^{T} \mathbf{P}\right) \mathbf{e}_{x}+\frac{\mathbf{e}_{x}^{T} \mathbf{P} \mathbf{P} \sigma}{\sigma}+\sigma \bar{C}^{2}\left\|\mathbf{e}_{x 2}\right\|^{2}+2 \mathbf{e}_{x}^{T} \mathbf{P} \bar{v} \\
& \leq \mathbf{e}_{x}^{T}\left(\mathbf{P A}+\mathbf{A}^{T} \mathbf{P}+\frac{\mathbf{P P}}{\sigma}+\varphi\right) \mathbf{e}_{x}+2 \mathbf{e}_{x}^{T} \mathbf{P} \bar{v} .
\end{aligned}
$$

If $Q=\mathbf{P A}+\mathbf{A}^{T} \mathbf{P}+(\mathbf{P P} / \sigma)+\varphi<0$ and $\Lambda=-\lambda_{\max }(\mathbf{Q})$, then we can get

$$
\begin{aligned}
\dot{V}_{1} \leq & -\Lambda\left\|\mathbf{e}_{x}\right\|^{2}-2 k_{1} \sum_{i=1}^{n} p_{1 i}\left|\mathbf{e}_{x 1 i}\right|-2 \mathbf{P}_{k 1} \mathbf{e}_{x}-2 k_{1} \mathbf{P}_{k 2} \mathbf{e}_{x} \\
& -2 k_{1}^{\gamma} \mathbf{P}_{k 3} \mathbf{e}_{x} \\
\leq & -\left\|\mathbf{e}_{x}\right\|\left(\begin{array}{c}
\Lambda\left\|\mathbf{e}_{x}\right\|-2 k_{1} \sqrt{\sum_{\mathbf{i}=1}^{\mathbf{n}} k_{2}^{2} p_{2 i}^{2}} \\
-2 k_{1}^{\gamma} \sqrt{\sum_{\mathbf{i}=1}^{\mathbf{n}} k_{3}^{2} p_{2 i}^{2}}-2 \sqrt{\sum_{\mathbf{i}=1}^{\mathbf{n}} p_{2 i}^{2} \chi_{i}^{2}}
\end{array}\right),
\end{aligned}
$$

where

$$
\left\{\begin{array}{l}
\mathbf{P}_{k 1}=\left[0_{1 \times n}, p_{21} \chi_{1} \operatorname{sign}\left(e_{11}\right), \ldots, p_{2 n} \chi_{n} \operatorname{sign}\left(e_{1 n}\right)\right], \\
\mathbf{P}_{k 2}=\left[0_{1 \times n}, p_{21} k_{2} \operatorname{sign}\left(e_{11}\right), \ldots, p_{2 n} k_{2} \operatorname{sign}\left(e_{1 n}\right)\right], \\
\mathbf{P}_{k 3}=\left[0_{1 \times n}, p_{21} k_{3} \operatorname{sign}\left(e_{11}\right), \ldots, p_{2 n} k_{3} \operatorname{sign}\left(e_{1 n}\right)\right] .
\end{array}\right.
$$

When $\left\|\mathbf{e}_{x}\right\|>R$,

$$
R=\frac{2 k_{1} \sqrt{\sum_{\mathbf{i}=\mathbf{1}}^{\mathbf{n}} k_{2}^{2} p_{2 i}^{2}}+2 k_{1}^{\gamma} \sqrt{\sum_{\mathbf{i}=\mathbf{1}}^{\mathbf{n}} k_{3}^{2} p_{2 i}^{2}}+2 \sqrt{\sum_{\mathbf{i}=\mathbf{1}}^{\mathbf{n}} p_{2 i}^{2} \boldsymbol{\chi}_{i}^{2}}}{\Lambda},
$$

and we can get $\dot{V}_{1}<0$. Therefore, when $\left\|\mathbf{e}_{x}\right\|$ exceeds its bound $R, \dot{V}_{1}<0$, while when $\left\|\mathbf{e}_{x}\right\|$ decreases, $\left\|\mathbf{e}_{x}\right\|$ decreases to its bounds $R$ within finite time.

Consider the second Lyapunov function as

$$
V_{2}=\frac{1}{2} \mathbf{e}_{x 1 \mathbf{i}}^{T} p_{1 i} \mathbf{e}_{x 1 \mathbf{i}} \text {. }
$$

Differentiating (30) with respect to time and substituting (23) into it yields

$$
\dot{V}_{2} \leq-p_{1 i}\left(l_{1 i}-t_{\mathbf{i}}\right) e_{x 1 \mathbf{i}}^{2}-p_{1 i}\left|\mathbf{e}_{x 1 \mathbf{i}}\right|\left(k_{1}-\left|\mathbf{e}_{x 2 \mathbf{i}}\right|\right)
$$

Let $l_{1 i}>t_{i}$ and $k_{1}-R=\eta>0$, (31) becomes

$$
\dot{V}_{2} \leq-p_{1 i} \eta\left|\mathbf{e}_{x 1 i}\right|=-\sqrt{2 p_{1 i}} \eta \dot{V}_{2}^{1 / 2}<0 .
$$

From (32), we can see that $\mathbf{e}_{x 1}$ and $\dot{e}_{x 1}$ can converge to zero in finite time.

When the sliding mode $\mathbf{e}_{x 1}=\dot{e}_{x 1}=0$, we have $\mathbf{v}_{1}=-\mathbf{e}_{x 2}$ and $\mathbf{v}_{2}=-k_{2} \mathbf{e}_{x 2}-k_{3}\left|\mathbf{e}_{x 2}\right|^{\gamma} \operatorname{sign}\left(e_{x 2}\right)$.

Take another Lyapunov function as

$$
V_{3}=\mathbf{e}_{x 2}^{T} \mathbf{P}_{2} \mathbf{e}_{x 2} .
$$

Differentiating (33) with respect to time and substituting (23) into it yields

$$
\begin{aligned}
\dot{V}_{3} \leq & \mathbf{e}_{2}^{T}\left(-2 \mathbf{P}_{2} \mathbf{T}+\frac{\mathbf{P}_{2} \mathbf{P}_{2}}{\sigma}+\mathbf{P}_{2} \bar{C}^{2} E\right) e_{x 2}-2 \sum_{\mathbf{i}=1}^{\mathbf{n}} \boldsymbol{\chi}_{i} p_{2 i}\left|\mathbf{e}_{x 2 i}\right| \\
& -2 \sum_{\mathbf{i}=1}^{\mathbf{n}} k_{2} p_{2 i} \mathbf{e}_{x 2 i}^{2}-2 \sum_{\mathbf{i}=1}^{\mathbf{n}} k_{3} p_{2 i}\left|\mathbf{e}_{x 2 i}\right|^{1+\gamma} .
\end{aligned}
$$

Since we have $-2 \mathbf{P}_{2} \mathbf{T}+\left(\mathbf{P}_{2} \mathbf{P}_{2} / \sigma\right)+\sigma \bar{C}^{2} \mathbf{E}<0$ according to Lemma 1 , we can further obtain

$$
\begin{aligned}
\dot{V}_{3} & \leq-2 \sum_{\mathbf{i}=1}^{\mathbf{n}} k_{2} p_{2 i} \mathbf{e}_{x 2 i}^{2}-2 \sum_{\mathbf{i}=1}^{\mathbf{n}} k_{2} p_{2 i}\left|\mathbf{e}_{x 2 \mathbf{i}}\right|^{1+\gamma} \\
& =-2 k_{2} V_{3}-2 k_{3} \sum_{\mathbf{i}=1}^{\mathbf{n}} p_{2 i}^{(1-\gamma) / 2} V_{3}^{(1+\gamma) / 2}<0 .
\end{aligned}
$$

From (35), we can see that $\mathbf{e}_{x 2}$ can converge to zero in finite time.

\subsection{Stability Analysis of the Closed-Loop System}

Lemma 2 (see [21]). If the control gain $\overline{\mathbf{M}}$ in (9) is chosen to satisfy the condition $\left\|\mathbf{I}-\mathbf{M}^{-1} \mathbf{q} \overline{\mathbf{M}}\right\|_{2}<1$ for all $t \geq 0$, then $\left\|\ddot{q}_{t-L}-\ddot{q}\right\|_{2} \longrightarrow 0$ as $L \longrightarrow 0$ and the error $\mathbf{H}-\widehat{\mathbf{H}}$ is bounded by a constant $H *$, i.e., $\|\mathbf{H}-\widehat{H}\| \leq H^{*}$. 
Lemma 3 (see [9]). For robotic system (1) controlled by the TDC with adaptive gradient estimators (14) and (15), the adaptive gain of the gradient $\widehat{K}_{\mathrm{GE}}$ has an upper bound:

$$
\left\|\widehat{K}_{\mathrm{GE}}\right\|<\left\|\widehat{K}_{\mathrm{GE}}^{*}\right\| \text {, }
$$

where $\widehat{K}_{G E}^{*}$ is a positive constant.

Theorem 1. Consider the robotic system (1) which is controlled by the TDC with gradient estimator (13) and the adaptive law (14). Then, the closed-loop system (15) is stable and the position tracking error $\mathbf{e}$ of the robotic system is bounded.

Proof. Define

$$
\mathbf{H} \approx \mathbf{H}_{t-L}=\widehat{H}=\ddot{q}_{t-L}-\bar{M}^{-1} \boldsymbol{\tau}_{t-L} .
$$

Now consider the Lyapunov function

$$
V=\frac{1}{2} \mathbf{S}^{T} \mathbf{S}+\frac{1}{2} \frac{\alpha}{\psi}\left[H^{*}-\widehat{K}_{\mathrm{GE}}\left(\dot{e}+\mathbf{K}_{D} \mathbf{e}+\mathbf{K}_{P} \int \mathbf{e} \mathbf{d t}\right)\right]^{2} .
$$

Let $\Delta=\dot{e}+\mathbf{K}_{D} \mathbf{e}+\mathbf{K}_{P} \int \mathbf{e} \mathbf{d t}$. Differentiating (38) and substituting $\Delta$ into it yields

$$
\dot{V}=\mathbf{S}^{T} \dot{S}-\frac{\alpha}{\psi}\left(H^{*}-\widehat{K}_{\mathrm{GE}} \Delta\right)\left(\dot{\hat{K}}_{\mathrm{GE}}+\dot{\Delta}\right) .
$$

From (8), we have $\dot{\Delta}=\varepsilon$. Now, substituting $\dot{S}$ and $\ddot{q}$ into (39), we can get

$$
\dot{V}=\mathbf{S}^{T}\left(\ddot{q}_{d}-\mathbf{H}-\bar{M}^{-1} \boldsymbol{\tau}+\mathrm{K}_{S} \dot{e}\right)-\frac{\alpha}{\psi}\left(H^{*}-\widehat{K}_{\mathrm{GE}} \Delta\right)\left(\dot{\hat{K}}_{\mathrm{GE}}+\varepsilon\right) .
$$

Substituting (15) and (37) into (39), we have

$$
\dot{V}=\mathbf{S}^{T}(-\mathbf{R}-\widehat{H}+\delta)-\frac{\alpha}{\psi}\left(H^{*}-\widehat{K}_{\mathrm{GE}} \Delta\right)\left(\dot{\hat{K}}_{\mathrm{GE}}+\varepsilon\right) \text {, }
$$

where $\mathbf{R}=\mathbf{H}-\widehat{H}$ and $\delta=-\bar{M}^{-1} \tau_{t-L}+\ddot{q}_{t-L}-\beta \mathbf{S}-\widehat{K}_{\mathrm{GE}} \Delta$.

Substituting (37) into (41), we can obtain

$$
\begin{aligned}
\dot{V} & =\mathbf{S}^{T}\left(-\mathbf{R}-\beta \mathbf{S}-\widehat{K}_{\mathrm{GE}} \Delta\right)-\frac{\alpha}{\psi}\left(H^{*}-\widehat{K}_{\mathrm{GE}} \Delta\right)\left(\dot{\hat{K}}_{\mathrm{GE}}+\varepsilon\right) \\
& \leq\|\mathbf{S}\|\|\mathbf{R}\|-\|\mathbf{S}\| \widehat{K}_{\mathrm{GE}} \Delta-\frac{\alpha}{\psi}\left(H^{*}-\widehat{K}_{\mathrm{GE}} \Delta\right)\left(\dot{\hat{K}}_{\mathrm{GE}}+\varepsilon\right)-\beta \mathbf{S}^{2} \\
& =\left[\|\mathbf{S}\|-\frac{\alpha}{\psi}(\dot{\hat{K}}+t \varepsilon)\right]\left(H^{*}-\widehat{K}_{\mathrm{GE}} \Delta\right)-\beta \mathbf{S}^{2} .
\end{aligned}
$$

Furthermore, $\Gamma$ is taken as the upper bound of $\mathbf{S}$, and according to the adaptive law (14), we have

$$
\dot{V} \leq-\beta \mathbf{S}^{2} \leq-\beta \Gamma^{2} .
$$

From (43), we have $\dot{V} \leq 0$.

According to (38), we can obtain

$$
\frac{1}{2} \mathbf{S}^{2} \leq V \leq \frac{1}{2} \mathbf{S}^{2}+\frac{1}{2} \frac{\alpha}{\psi}\left[H^{*}-\widehat{K}_{\mathrm{GE}}\left(\dot{e}+\mathbf{K}_{D} \mathbf{e}+\mathbf{K}_{P} \int \mathbf{e d t}\right)\right]^{2}
$$

Since $H^{*}$ is a constant, from Lemma 2, it can be known that $\widehat{K}_{\mathrm{GE}}$ is bounded. Now let $Z$ be the bound of $(1 / 2)(\alpha / \psi)\left[H^{*}-\widehat{K}_{\mathrm{GE}}\left(\dot{e}+t \mathbf{K}_{D} n e q+h \mathbf{K}_{P} \mathbf{e} \mathbf{d t}\right]^{2}\right.$ in $(44)$ and we can get

$$
V<\frac{1}{2} \Gamma^{2}+\frac{1}{2} Z^{2}
$$

According to (44) and (45), we can obtain

$$
\frac{1}{2} \mathbf{S}^{2}<\frac{1}{2} \Gamma^{2}+\frac{1}{2} \mathrm{Z}^{2}
$$

Thus, we can further get

$$
\|\mathbf{S}\|<\sqrt{\Gamma^{2}+Z^{2}} .
$$

Equations (44) and (46) show that $\mathbf{S}$ has an upper bound and can be adjusted by $\alpha, \psi$, and $\Gamma$. Since $\mathbf{S}$ is bounded and $\mathbf{S}=\dot{e}+\mathbf{K}_{S} \mathbf{e}, \dot{e}=-\mathbf{K}_{S} \mathbf{e}$ is asymptotically stable. The position tracking error e of the robotic system is bounded. This concludes the proof of Theorem 1 .

\section{Simulation Studies}

In order to verify the effectiveness of the velocity-free adaptive TDC, simulations are conducted on a 2-DOF robot and compared to the conventional TDC in Section 2 and the adaptive gain sliding mode TDC in [9].

The dynamics of the robot is as follows:

$$
\begin{aligned}
& \mathbf{M}=\left[\begin{array}{cc}
\left(m_{1}+m_{2}\right) l_{1}^{2}+m_{2} l_{2}^{2}+2 m_{2} l_{1} l_{2} \cos \mathbf{q}_{2} & m_{2} l_{2}^{2} \cos \mathbf{q}_{2} \\
m_{2} l_{2}^{2} \cos \mathbf{q}_{2} & m_{2} l_{2}^{2}
\end{array}\right], \\
& \mathbf{C}=\left[\begin{array}{cc}
-m_{2} l_{1} l_{2} \dot{q}_{2} \sin \mathbf{q}_{2} & -m_{2} l_{1} l_{2} \dot{q}_{1} \dot{q}_{2} \sin \mathbf{q}_{2} \\
m_{2} l_{1} l_{2} \dot{q}_{1} \sin \mathbf{q}_{2} & 0
\end{array}\right], \\
& \mathbf{G}=\left[\begin{array}{c}
\left.\left[m_{1}+m_{2}\right) l_{1} \cos \mathbf{q}_{2}+m_{2} l_{2} \cos \left(\mathbf{q}_{1}+\mathbf{q}_{2}\right)\right] g \\
m_{2} l_{2} \cos \left(\mathbf{q}_{1}+\mathbf{q}_{2}\right) g
\end{array}\right], \\
& \mathbf{f}=0.02 \operatorname{sign}(\dot{q}),
\end{aligned}
$$

where $m_{1}$ and $m_{2}$ represent the mass of links, $l_{1}$ and $l_{2}$ are the length of links, and $g$ is the gravitational acceleration, respectively.

In the simulations, $m_{1}=m_{2}=0.5, l_{1}=1, l_{2}=0.8$, and $g=9.8$. The desired positions of joint 1 and joint 2 of the robot are $q_{d 1}=q_{d 2}=\sin 2 \pi \mathrm{t}$. The time delay is set to be the sampling time, i.e., $L=0.001 \mathrm{~s}$.

When the conventional TDC (6) is employed, $\mathbf{K}_{D}=\operatorname{di} \mathbf{a g}\{80,60\}, \quad \mathbf{K}_{P}=\operatorname{di} \mathbf{a g}\{200,180\}, \quad$ and $\bar{M}=$ diag $\{0.5,0.4\}$. The simulation results of the conventional TDC are shown in Figures 2-4.

From Figure 2, it can be seen that, with the conventional TDC, the real position trajectory of joint 1 and joint 2 of the robot cannot track the desired position in a satisfactory way. 


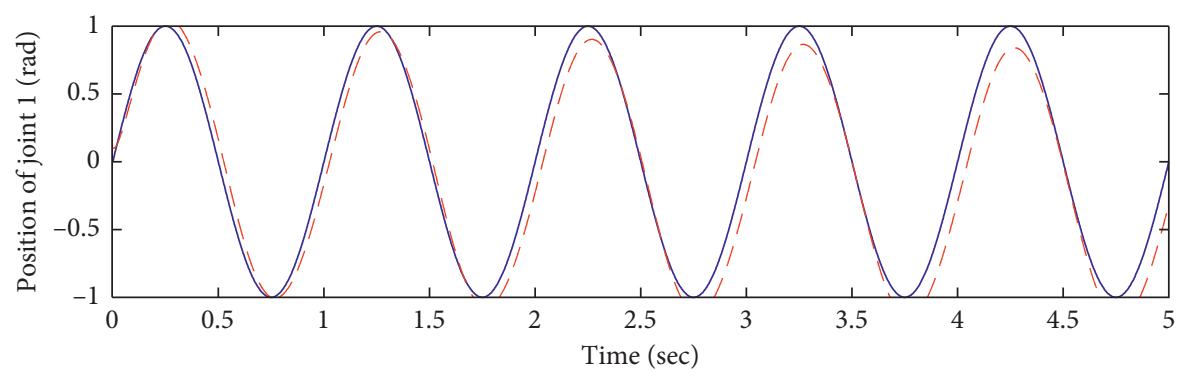

— Desired position

- - - Real position

(a)

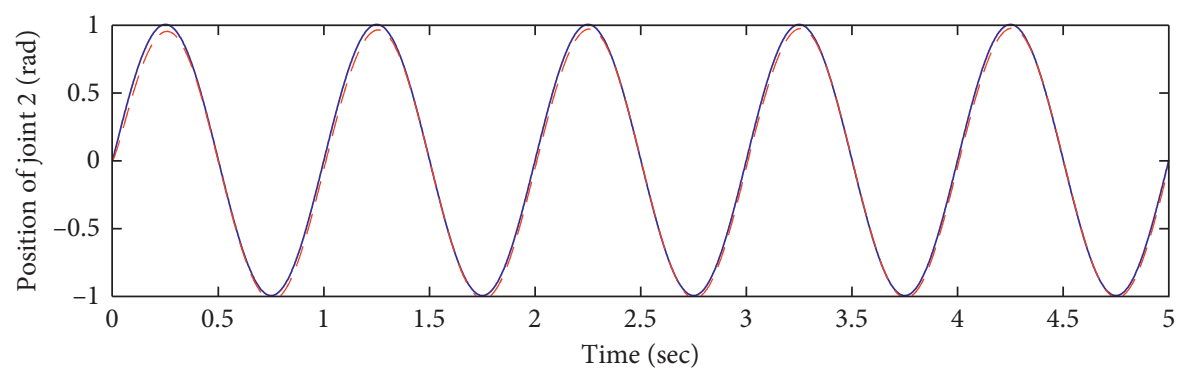

- Desired position

- - Real position

(b)

Figure 2: Position tracking of (a) joint 1 and (b) joint 2 (conventional TDC).

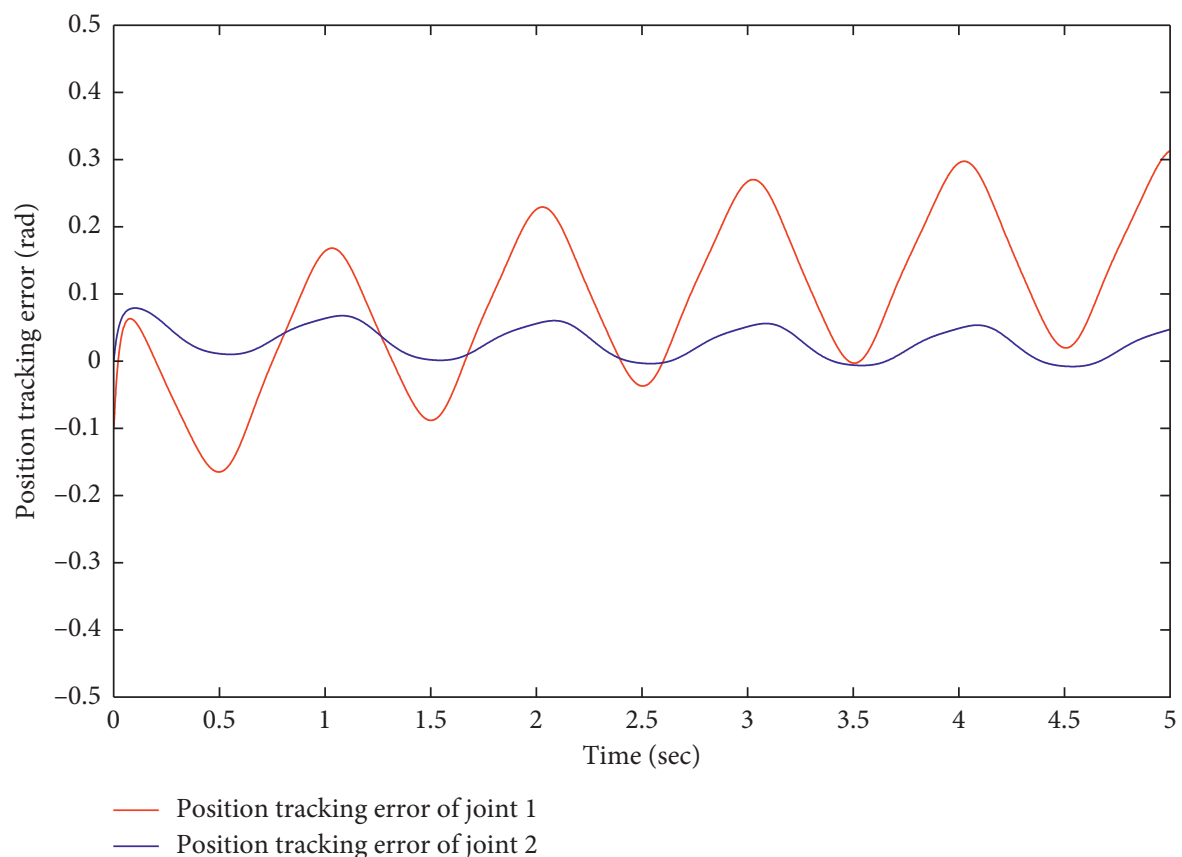

FIgURe 3: Position tracking error of joint 1 and joint 2 (conventional TDC).

There exists an obvious position error, especially for joint 1 , as shown in Figure 3. Besides, as shown in Figure 4, the fluctuation of the input torque is obvious especially when it achieves the maximum value each time.
When the adaptive gain sliding mode TDC in [9] is employed, $\mathbf{K}_{S}=\operatorname{di} \mathbf{a g}\{5,5\}, \beta=85, \alpha=20, \psi=0.001$, and $\bar{M}=$ di ag $\{1,0.9\}$. The simulation results are shown in Figures 5-7. 


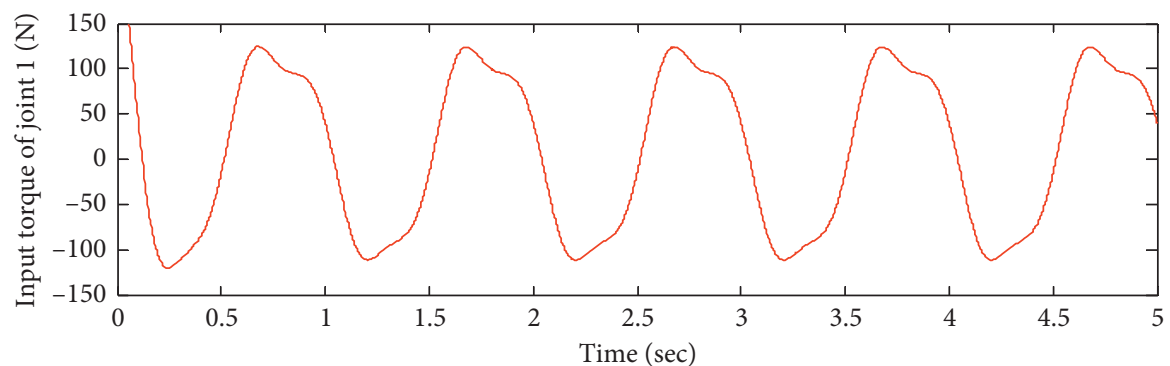

(a)

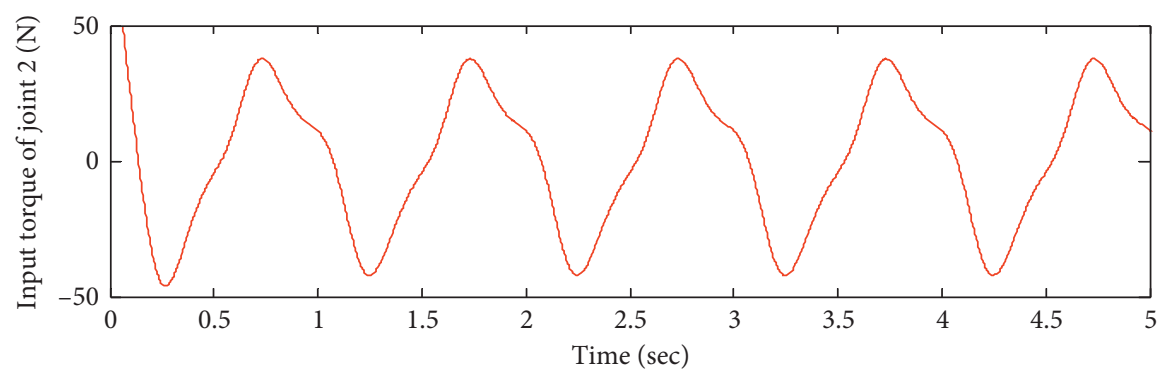

(b)

FIgURE 4: Input torque of (a) joint 1 and (b) joint 2 (conventional TDC).

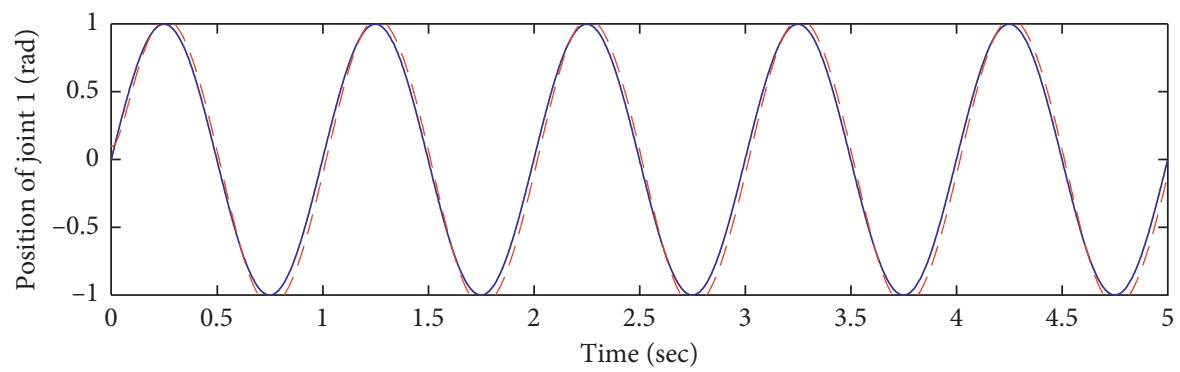

_ Desired position

- - - Real position

(a)

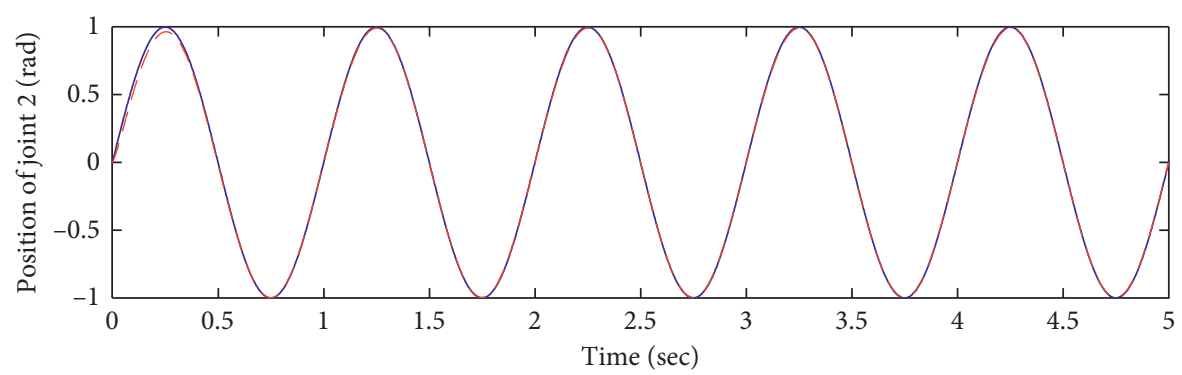

- Desired position

-. Real position

(b)

FIgURE 5: Position tracking of (a) joint 1 and (b) joint 2 (adaptive gain sliding mode TDC). 


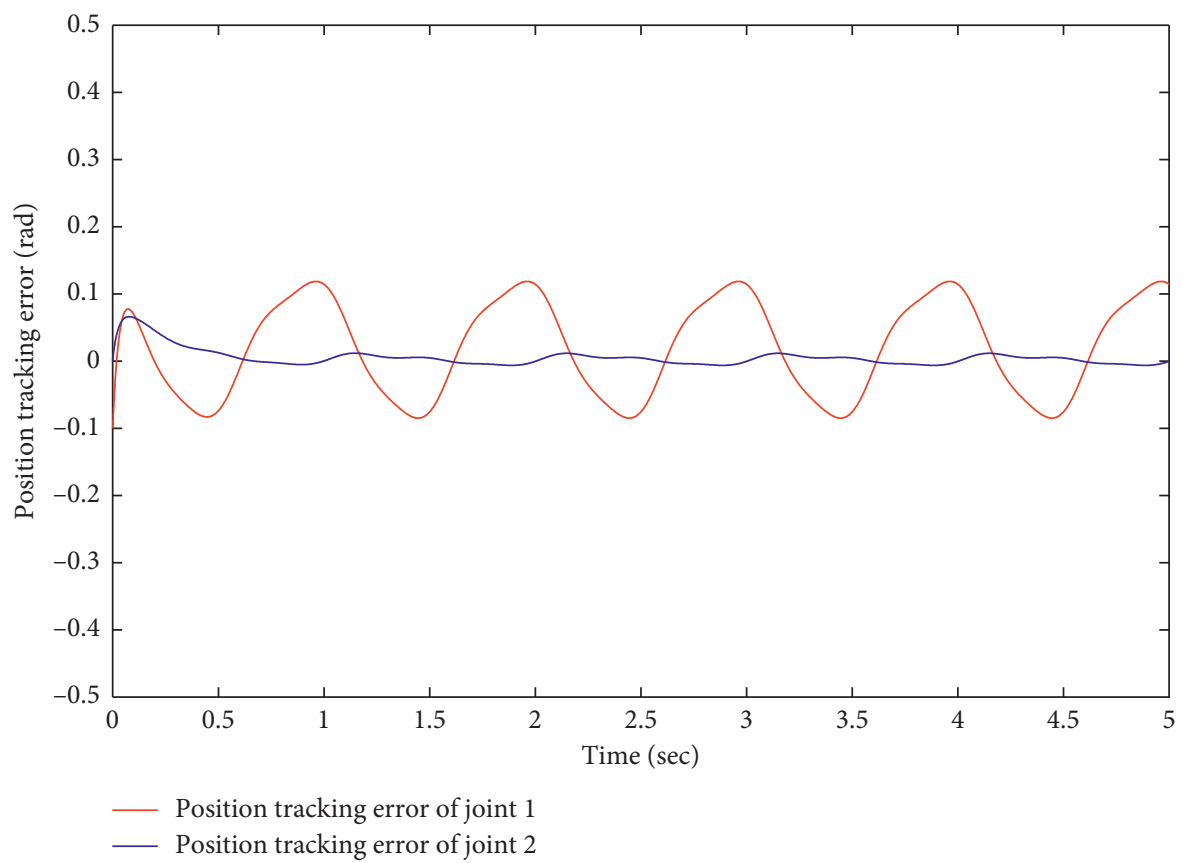

FIgURE 6: Position tracking error of joint 1 and joint 2 (adaptive gain sliding mode TDC).

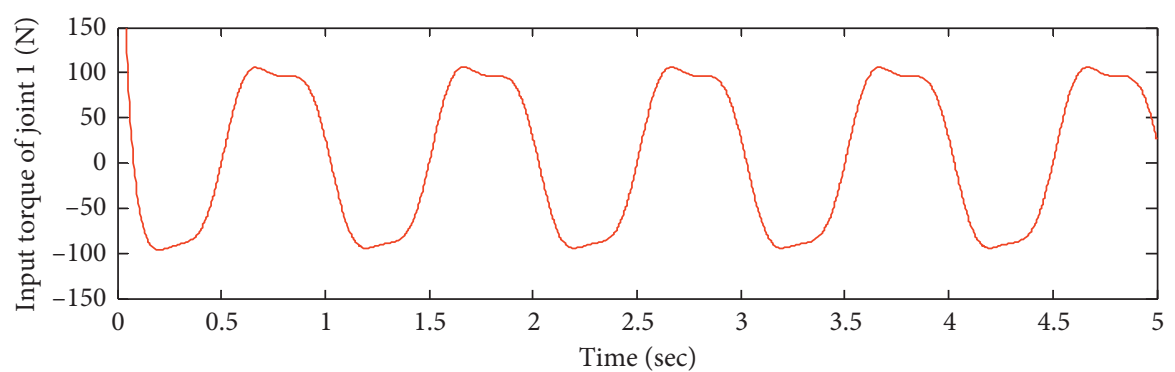

(a)

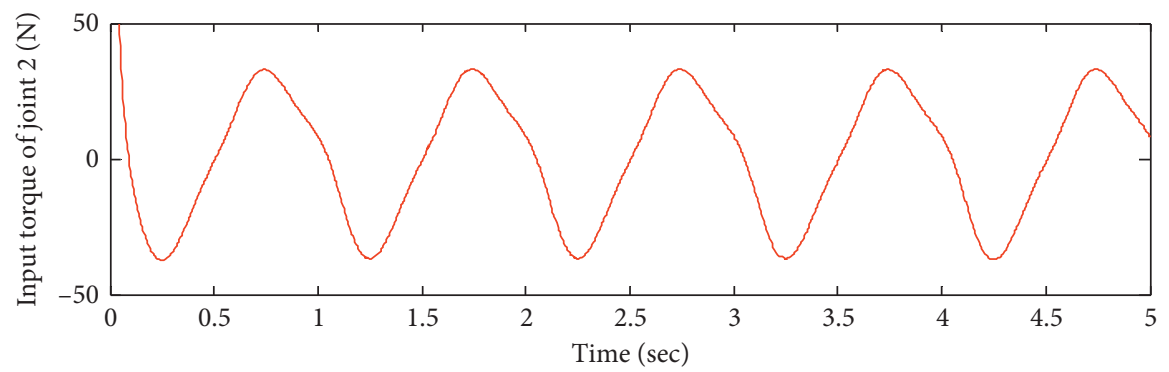

(b)

FIgURE 7: Input torque of (a) joint 1 and (b) joint 2 (adaptive gain sliding mode TDC).

From Figure 5, it can be seen that, with the adaptive gain sliding mode TDC, the position tracking of joint 1 has better tracking performance than that of the conventional TDC. However, the real position trajectory of joint 1 and joint 2 of the robot cannot track the desired position in a satisfying way either. There still exists obvious position error, especially for joint 1 , as shown in Figure 6. In addition, the fluctuation of the input torque still exists, as shown in Figure 7, although it is smaller than that of the conventional TDC.
When the proposed control is employed, $\beta=20$, $\mathbf{K}_{S}=\operatorname{di} \mathbf{a g}\{5,10\}, \quad \bar{M}=\operatorname{di} \mathbf{a g}\{0.05,0.04\}, \quad \alpha=20, \quad$ and $\psi=0.001$. The initial value of the gain of the gradient is $\widehat{K}_{\mathrm{GE}}(0)=\operatorname{di} \mathbf{a g}\left\{K_{\mathrm{GE}_{1}}(0), K_{\mathrm{GE}_{2}}(0)\right\}, \quad K_{\mathrm{GE}_{1}}(0)=0, \quad$ and $K_{\mathrm{GE}_{2}}(0)=0$. For the observer, $\mathbf{T}=\mathbf{d i} \mathbf{a g}\{0.01,0.1\}$, $\mathbf{L}_{1}=\operatorname{di} \mathbf{a g}\{1,1\}, \quad \mathbf{L}_{2}=\operatorname{di} \mathbf{a g}\{0.001,0.001\}, \quad \chi=\operatorname{diag}\{0.0001$, $0.0001\}, k_{1}=0.0001, k_{2}=1, k_{3}=3$, and $\gamma=0.5$. The simulation results of the proposed control are shown in Figures 8-13. 


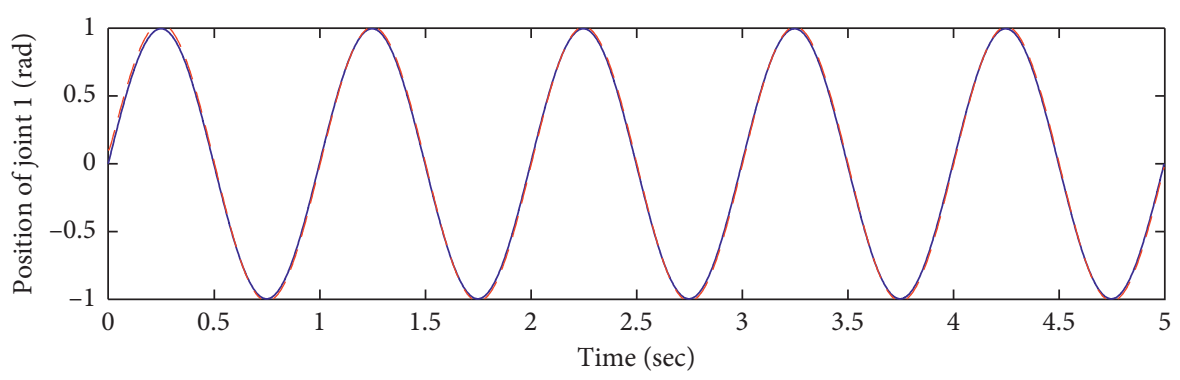

- Desired position

- - Real position

(a)

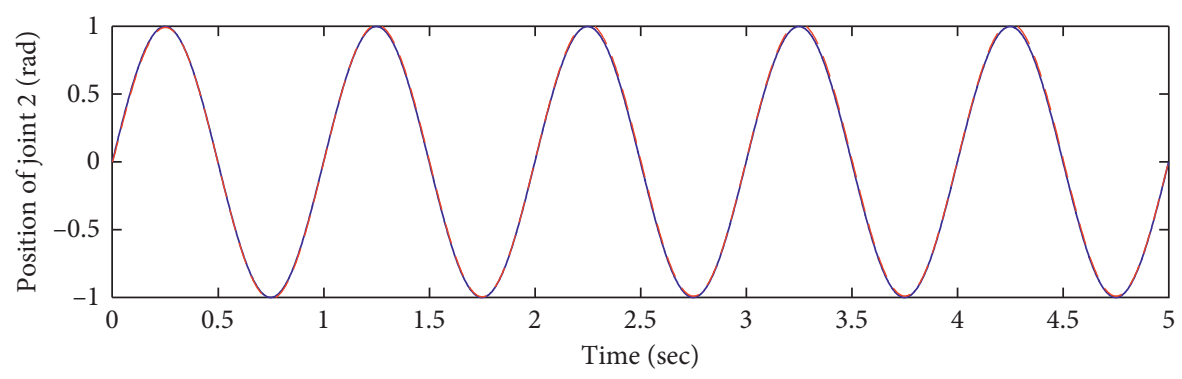

- Desired position

- - - Real position

(b)

Figure 8: Position tracking of (a) joint 1 and (b) joint 2 (proposed control).

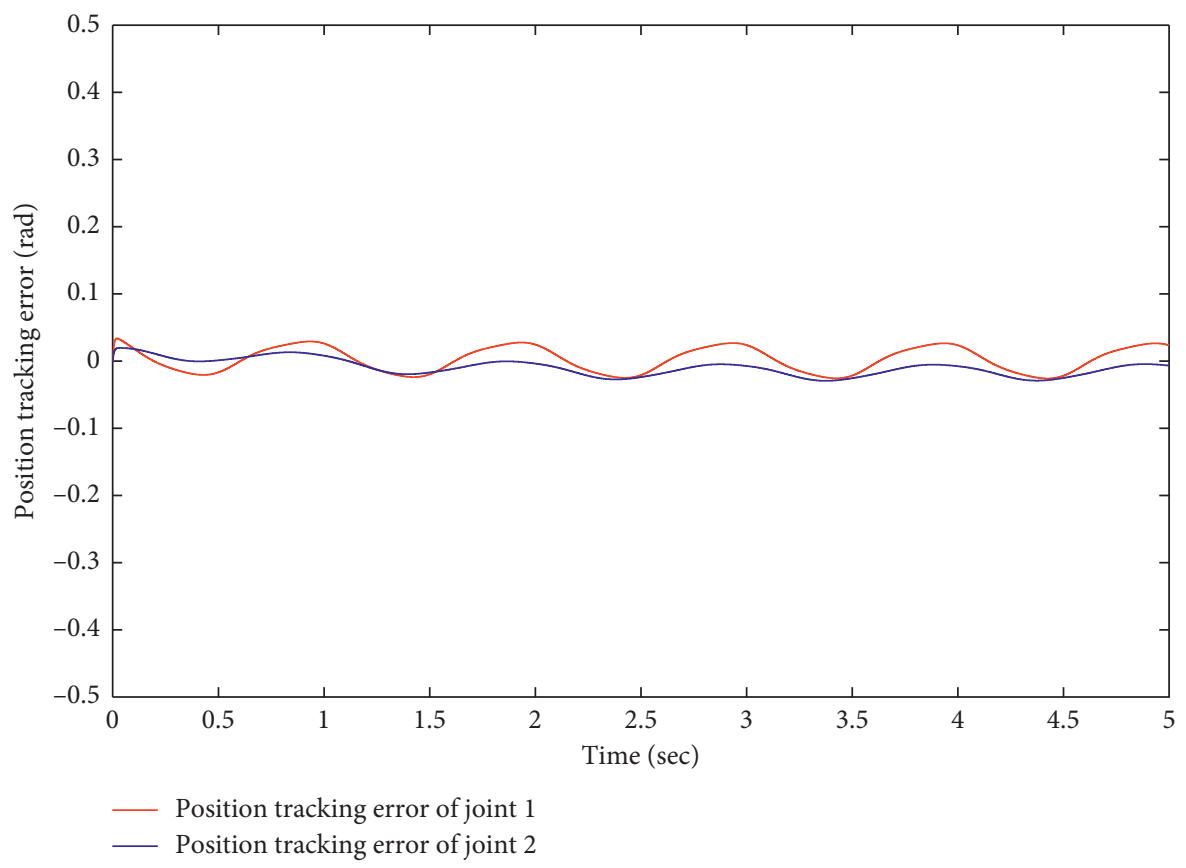

FIgure 9: Position tracking error of joint 1 and joint 2 (proposed control).

From Figure 8, it can be seen that the real position trajectory of joint 1 and joint 2 of the robot can track the desired position in a satisfying way. The position tracking errors of both joints 1 and 2 are quite small, as shown in
Figure 9. Moreover, from Figure 10, the fluctuation of the input torque is the smallest compared with that of the conventional TDC and the adaptive gain sliding mode TDC. Figure 11 shows that the gain of the gradient can be adjusted 


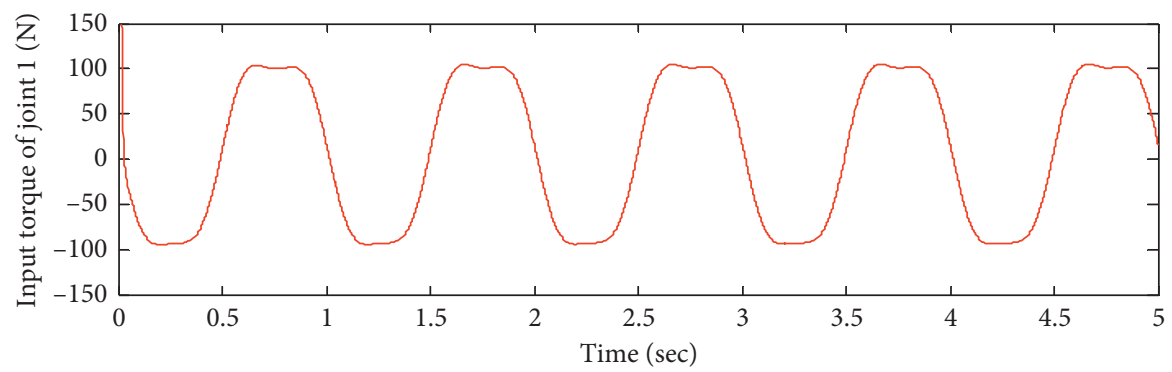

(a)

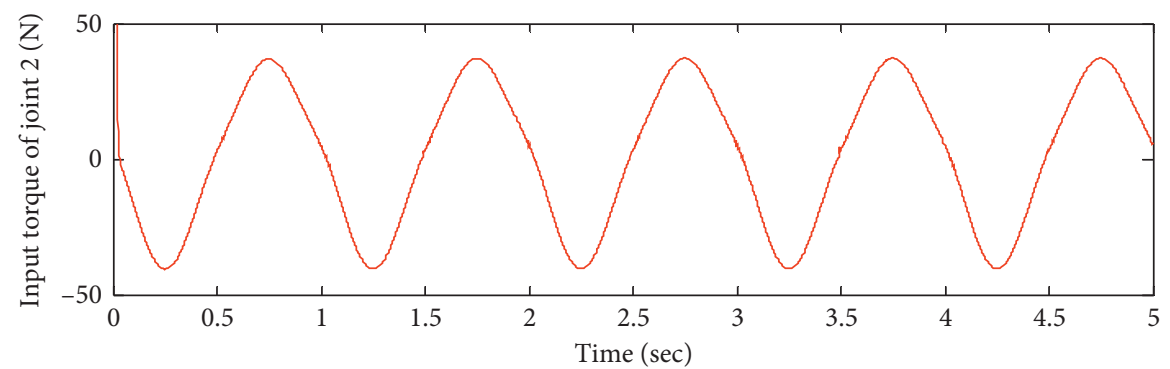

(b)

FIgURE 10: Input torque of (a) joint 1 and (b) joint 2 (proposed control).

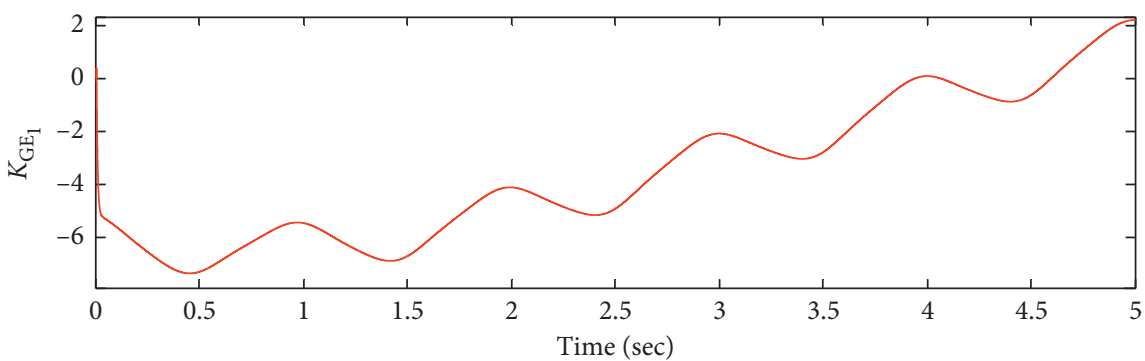

(a)

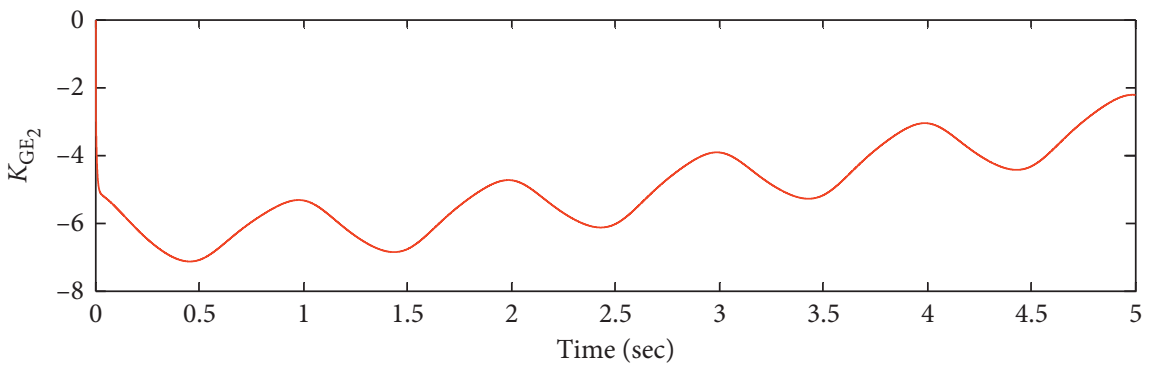

(b)

FIGURE 11: Adaptive gain of the gradient (a) $K_{\mathrm{GE}_{1}}$ and (b) $K_{G_{2}}$ (proposed control).

by the adaptive law. From Figures 12 and 13, it can be seen that the observer can effectively observe the velocity and acceleration of the robot.

In order to quantitatively compare the position tracking performance of the proposed method with the conventional TDC method and the adaptive gain sliding mode TDC in [9], the range of the position tracking error $e_{r_{n}}=$ $\left|e_{\min _{n}}\right| \sim\left|e_{\max _{n}}\right|$ is calculated, where $n=1,2$ represents joint 1 and joint 2 , respectively. The calculation results are shown in Table 1.
From Table 1, it can be seen that compared to the conventional TDC, the maximum position tracking error of joint 1 is $0.279362 \mathrm{rad}$ smaller and that of joint 2 is $0.04987 \mathrm{rad}$ smaller with the proposed control. In addition, compared to the adaptive gain sliding mode TDC, the maximum position tracking error of joint 1 is 0.08476 rad smaller and that of joint 2 is $0.03697 \mathrm{rad}$ smaller with the proposed control.

Remark 4. There are three parameters to be chosen in the TDC with adaptive gradient estimator, i.e., $\alpha, \beta$, and $\psi$. In 


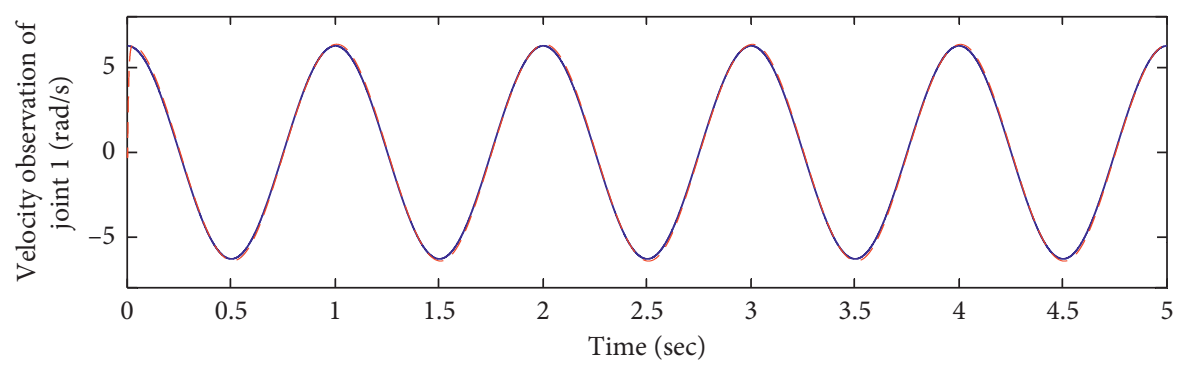

- Real velocity

- - Velocity observation

(a)

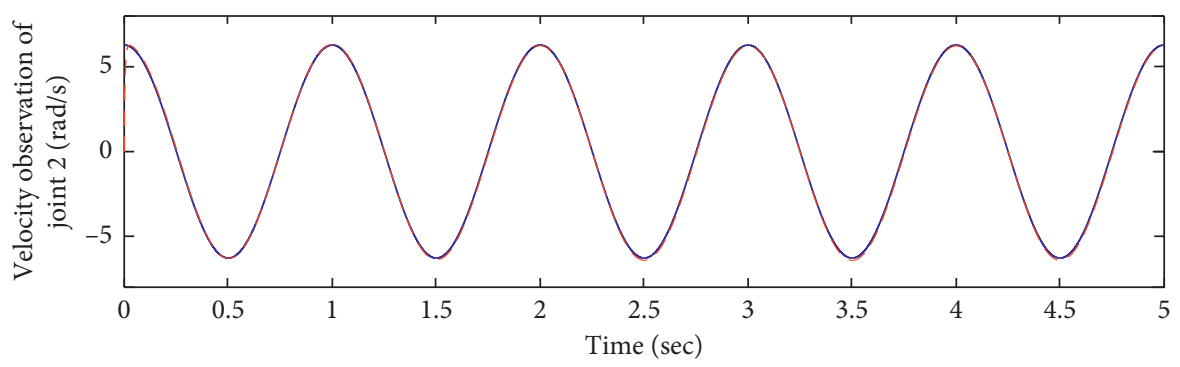

Real velocity

- - Velocity observation

(b)

FIgURE 12: Velocity observation of (a) joint 1 and (b) joint 2 (proposed control).

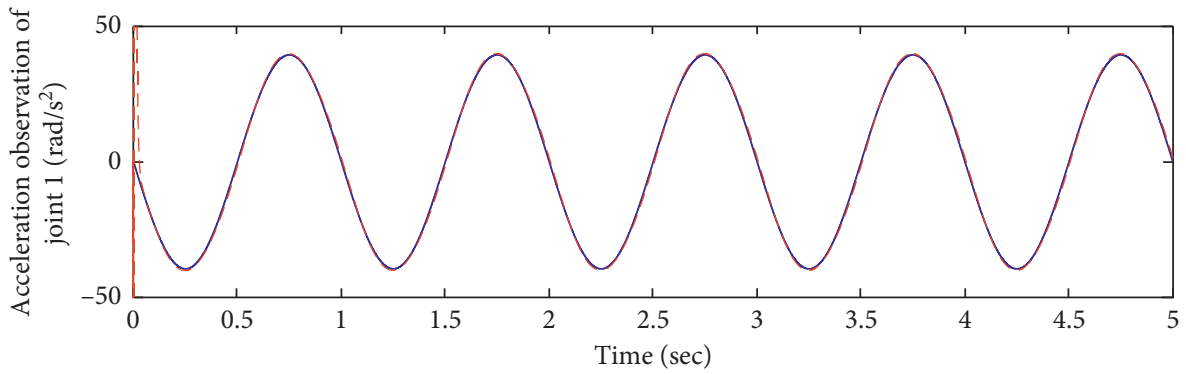

- Real acceleration

- - - Acceleration observation

(a)

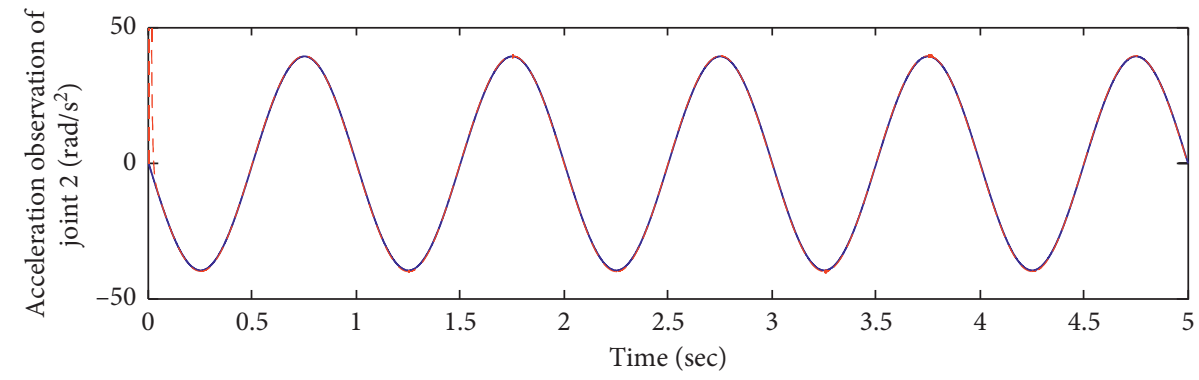

- - - Acceleration observation

(b)

FIgURe 13: Acceleration observation of (a) joint 1 and (b) joint 2 (proposed control). 
TABLE 1: Quantitative comparison of position tracking error.

\begin{tabular}{lccc}
\hline & Conventional TDC & Adaptive gain sliding mode TDC & Proposed control \\
\hline$e_{r_{1}}(\mathrm{rad})$ & $0-0.3133$ & $0-0.1187$ & $0-0.03394$ \\
$e_{r_{2}}(\mathrm{rad})$ & $0-0.0790$ & $0-0.0661$ & $0-0.02913$ \\
\hline
\end{tabular}

addition, there are four parameters to be chosen in the observer, i.e., $k_{1}, k_{2}, k_{3}$, and $\gamma$. When $\alpha, \beta, \psi, k_{1}, k_{2}, k_{3}$, and $\gamma$ increase to ten times of their values (or decrease to ten times of their values), the position tracking errors of joint 1 and joint 2 will become bigger.

\section{Conclusions}

To improve the trajectory tracking performance of complex nonlinear robotic system, this paper proposes a velocity-free adaptive TDC. A TDC with gradient estimator is designed considering that conventional TDC may cause large TDE error. Then, an adaptive law is designed to estimate the gain of the gradient. Next, an observer is designed to observe the velocity and acceleration in the controller. The proposed control can not only offset the nonlinear terms in the complex dynamics of the robotic system but also reduce the TDE error, estimate the gain of the gradient online, and avoid the measurement of velocity and acceleration. Experimental verification of the control proposed in this paper is quite necessary and remains as our work in the next step.

\section{Data Availability}

The data that supports our manuscript conclusions are some open access articles that have been properly cited, and the readers can easily obtain these articles to verify the conclusions.

\section{Conflicts of Interest}

The authors declare that they have no conflicts of interest regarding the publication of this paper.

\section{Acknowledgments}

This research was supported by the National Natural Science Foundation of China (Grant nos. 61973257, 61973331, and 61875166), Sichuan Science and Technology Program (Grant no. 2020YJ0367), and the Innovation Fund Project of Xihua University for Graduate Students (Grant no. YCJJ2020049).

\section{References}

[1] L. Zhang, Z. Li, and C. Yang, "Adaptive neural network based variable stiffness control of uncertain robotic systems using disturbance observer," IEEE Transactions on Industrial Electronics, vol. 64, no. 3, pp. 2236-2245, 2017.

[2] Z. Liu, G. Lai, Y. Zhang, and C. L. P. Chen, "Adaptive fuzzy tracking control of nonlinear time-delay systems with deadzone output mechanism based on a novel smooth model," IEEE Transactions on Fuzzy Systems, vol. 23, no. 6, pp. 1998-2011, 2015.
[3] R. Babaghasabha, M. A. Khosravi, and H. D. Taghirad, "Adaptive robust control of fully-constrained cable driven parallel robots," Mechatronics, vol. 25, pp. 27-36, 2015.

[4] K. Youcef-Toumi and O. Ito, "A time delay controller for systems with unknown dynamics," in Proceedings of the 1988 American Control Conference, pp. 194-208, Atlanta, GA, USA, June 1990.

[5] M. Jin, Y. Jin, P. H. Chang, and C. Choi, "High-accuracy tracking control of robot manipulators using time delay estimation and terminal sliding mode," International Journal of Advanced Robotic Systems, vol. 8, pp. 65-78, 2011.

[6] Z. Yang, J. Peng, and Y. Liu, "Adaptive neural network force tracking impedance control for uncertain robotic manipulator based on nonlinear velocity observer," Neurocomputing, vol. 331, pp. 263-280, 2019.

[7] M. Rahmani and K. Behdinan, "Interaction of torque link free play and Coulomb friction nonlinearities in nose landing gear shimmy scenarios," International Journal of Non-Linear Mechanics, vol. 119, Article ID 103338, 2020.

[8] M. Ruderman and M. Iwasaki, "Observer of nonlinear friction dynamics for motion control," IEEE Transactions on Industrial Electronics, vol. 62, no. 9, pp. 5941-5949, 2015.

[9] J. Baek, M. Jin, and S. Han, "A new adaptive sliding-mode control scheme for application to robot manipulators," IEEE Transactions on Industrial Electronics, vol. 63, no. 6, pp. 3628-3637, 2016.

[10] J. Baek, W. Kwon, B. Kim, and S. Han, "A widely adaptive timedelayed control and its application to robot manipulators," IEEE Transactions on Industrial Electronics, vol. 66, no. 7, pp. 5332-5342, 2019.

[11] Y. Wang, F. Yan, J. Chen, F. Ju, and B. Chen, "A new adaptive time-delay control scheme for cable-driven manipulators," IEEE Transactions on Industrial Informatics, vol. 15, no. 6, pp. 3469-3481, 2019.

[12] M. Jin, S. H. Kang, and P. H. Chang, "Robust compliant motion control of robot with nonlinear friction using timedelay estimation," IEEE Transaction on Industrial Electronics, vol. 55, no. 1, pp. 258-269, 2008.

[13] M. Jin, S. H. Kang, P. H. Chang, and J. Lee, "Robust control of robot manipulators using inclusive and enhanced time delay control," IEEE/ASME Transactions on Mechatronics, vol. 22, no. 5, pp. 2141-2152, 2017.

[14] G. R. Cho, P. H. Chang, S. H. Park, and M. Jin, "Robust tracking under nonlinear friction using time-delay control with internal model," IEEE Transactions on Control Systems Technology, vol. 17, no. 6, pp. 1406-1414, 2009.

[15] S. R. Jiang, Y. Y. Wang, F. Ju, B. Chen, and H. T. Wu, "A new fuzzy time-delay control for cable-driven robot," International Journal of Advanced Robotic Systems, vol. 16, no. 2, pp. 1-10, 2019.

[16] D. K. Han and P.-h. Chang, "Robust tracking of robot manipulator with nonlinear friction using time delay control with gradient estimator," Journal of Mechanical Science and Technology, vol. 24, no. 8, pp. 1743-1752, 2010.

[17] H. Yan, C. Sun, and G. Hu, "Robust tracking control of an Euler-Lagrange system subject to uncertain friction and impact," in Proceedings of the IEEE International Conference 
on Information and Automation, pp. 2227-2232, Lijiang, China, August 2015.

[18] H.-J. Bae, M. Jin, J. Suh, J. Y. Lee, P.-H. Chang, and D.-s. Ahn, "Control of robot manipulators using time-delay estimation and fuzzy logic systems," Journal of Electrical Engineering and Technology, vol. 12, no. 3, pp. 1271-1279, 2017.

[19] S. Ahmad, M. Rehan, and M. Iqbal, "Robust generalized filtering of uncertain Lipschitz nonlinear systems under measurement delays," Nonlinear Dynamics, vol. 92, no. 4, pp. 1567-1582, 2018.

[20] C. Hua, Y. Yang, and P. X. Liu, "Output-feedback adaptive control of networked teleoperation system with time-varying delay and bounded inputs," IEEE/ASME Transactions on Mechatronics, vol. 20, no. 5, pp. 2009-2020, 2015.

[21] M. Jin, J. Lee, P. H. Chang, and C. Choi, "Practical nonsingular terminal sliding-mode control of robot manipulators for high-accuracy tracking control," IEEE Transactions on Industrial Electronics, vol. 56, no. 9, pp. 3593-3601, 2009. 